\title{
Double-layer graphene for enhanced tunable infrared plasmonics
}

\author{
Daniel Rodrigo ${ }^{1}$, Andreas Tittl ${ }^{1}$, Odeta Limaj ${ }^{1}$, F Javier García de Abajo ${ }^{2,3}$, Valerio Pruneri ${ }^{2,3}$ \\ and Hatice Altug ${ }^{1}$
}

Graphene is emerging as a promising material for photonic applications owing to its unique optoelectronic properties. Graphene supports tunable, long-lived and extremely confined plasmons that have great potential for applications such as biosensing and optical communications. However, in order to excite plasmonic resonances in graphene, this material requires a high doping level, which is challenging to achieve without degrading carrier mobility and stability. Here, we demonstrate that the infrared plasmonic response of a graphene multilayer stack is analogous to that of a highly doped single layer of graphene, preserving mobility and supporting plasmonic resonances with higher oscillator strength than previously explored single-layer devices. Particularly, we find that the optically equivalent carrier density in multilayer graphene is larger than the sum of those in the individual layers. Furthermore, electrostatic biasing in multilayer graphene is enhanced with respect to single layer due to the redistribution of carriers over different layers, thus extending the spectral tuning range of the plasmonic structure. The superior effective doping and improved tunability of multilayer graphene stacks should enable a plethora of future infrared plasmonic devices with high optical performance and wide tunability.

Light: Science \& Applications (2017) 6, e16277; doi:10.1038/Isa.2016.277; published online 2 June 2017

Keywords: graphene; infrared; nanophotonics; optoelectronic; plasmonics

\section{INTRODUCTION}

Plasmons are optically driven collective electron oscillations capable of concentrating light down to deeply subwavelength volumes ${ }^{1,2}$, laying the foundation for a multitude of essential applications in fields such as sensing ${ }^{3,4}$, light-harvesting ${ }^{5,6}$ and photochemistry ${ }^{7}$. While commonly used noble metals such as silver and gold exhibit plasmonic properties mostly in the visible and near-infrared region of the spectrum, graphene has recently emerged as a new material able to extend the field of plasmonics to infrared and terahertz wavelengths ${ }^{8-13}$. Localized surface plasmon resonances can be excited in graphene by patterning it into optically resonant nanostructures, such as nanoribbons ${ }^{14-20}$ or nanodisks ${ }^{21-23}$. In contrast to noble metals, graphene plasmonic resonances can be dynamically tuned through electrostatic biasing ${ }^{24,25}$ and enable a new generation of reconfigurable plasmonic devices ${ }^{26-30}$. In addition, plasmons excited in graphene can reach remarkably long lifetimes and provide unprecedented levels of light confinement ${ }^{31}$. Owing to these unique electro-optical properties, there has been a strong focus on developing new graphene-based plasmonic devices, and in particular infrared biosensors ${ }^{32}$, modulators ${ }^{33-35}$, metasurfaces ${ }^{36-38}$ and perfect absorbers $^{39,40}$.

In single-layer graphene (SLG), plasmonic resonances possess relatively low oscillator strengths because the attainable carrier densities are low. In the pursuit of stronger resonances, improved carrier mobility and new capabilities, the exploration of graphene plasmons in stack-ups composed of graphene and other twodimensional (2D) materials has recently attracted significant interest. Initially, multilayer graphene (MLG) was investigated in the terahertz range and was shown to provide plasmonic resonances with larger amplitude modulation ${ }^{15}$. Extending this concept to infrared frequencies, more recent work has focused on bilayer graphene $e^{41,42}$ and heterostructures composed of graphene on boron nitride ${ }^{31,43}$. In addition, MLG structures have been shown to exhibit resonances with higher infrared transmission modulation compared to $\mathrm{SLG}^{44,45}$. However, a satisfactory understanding of MLG, especially with regard to electrostatic biasing, is still lacking, thus limiting its application to actual devices. In this paper, we present a complete framework for understanding localized surface plasmon resonances in MLG stacks. In this multilayer structure, there is negligible interlayer electronic coupling and, unlike in graphite, the graphene band structure is retained. We explore the properties of MLG plasmonic nanoribbons, including spectral, near-field and tuning properties, and we show larger plasmonic enhancement associated with the multilayer geometry compared with previous results ${ }^{44}$. In particular, we show that double-layer graphene (DLG) provides wider spectral tuning ranges than SLG, in addition to sustaining resonances at higher frequencies and with higher extinction. Our analysis reveals that plasmon resonances in MLG can be quantitatively understood in the context

${ }^{1}$ Institute of BioEngineering, École Polytechnique Fédérale de Lausanne, CH-1015 Lausanne, Switzerland; ${ }^{2}$ ICFO_Institut de Ciències Fotòniques, The Barcelona Institute of Science and Technology, 08860 Castelldefels (Barcelona), Spain and ${ }^{3}$ ICREA—Institució Catalana de Recerca i Estudis Avançats, 08010 Barcelona, Spain

Correspondence: H Altug, Email: hatice.altug@epfl.ch

Received 19 April 2016; revised 3 January 2017; accepted 5 January 2017; accepted article preview online 9 January 2017 
of plasmon hybridization and provides a theoretical basis for the experimental evidence that the plasmonic response of DLG is equivalent to that of a SLG at a higher doping. Finally, we focus on the response of MLG to electrostatic biasing that demonstrate for the first time that the plasmonic effects of biasing are enhanced in MLG compared with SLG. Our results and proposed technique hold promise for a wide range of applications, as the performance of graphene-based photonic devices can be significantly improved by transferring two or more graphene layers rather than one.

\section{MATERIALS AND METHODS}

\section{Fabrication and characterization}

Graphene nanoribbon arrays are fabricated on a double-sided polished float-zone silicon substrate. A native oxide layer of $280 \mathrm{~nm}$ is grown by dry oxidation. Chemical vapor deposition (CVD)-grown graphene is wet-transferred over the oxide layer. DLG devices are formed by two successive transfers of SLG. Graphene nanoribbons are patterned with $100-\mathrm{keV}$ electron beam lithography and are etched in oxygen plasma. The transmission spectra of the graphene arrays are acquired with a Fourier-transform infrared spectrometer coupled to an infrared microscope (Bruker Vertex $80 \mathrm{~V}$ and Hyperion 3000). An infrared polarizer is used to select the polarization transversal to the graphene nanoribbons. Measurements are performed in a dry-air-purged atmosphere with a non-immersion Cassegrain objective and a mercurycadmium-tellurium detector cooled in liquid nitrogen.

\section{Numerical simulations}

The extinction spectra and charge distributions of the graphene nanoribbon arrays are calculated with a frequency-domain solver of Maxwell equations based on the finite elements method (commercial implementation Ansys HFSS). The periodic ribbon structure is simulated using Floquet-Bloch periodic boundary conditions. The convergence of simulations is ensured by monitorizing the transmission coefficient while refining the meshing in an iterative process. Graphene is modeled as a 2D layer with complex surface conductivity calculated from the Drude model: $\sigma=\frac{i e^{2} E_{\mathrm{F}}}{\hbar \pi(\omega+i / \tau)}$, where the Fermi level $E_{\mathrm{F}}$ accounts for room temperature effects: $E_{\mathrm{F}}=E_{\mathrm{F}, \mathrm{T}=0}+$ $2 k_{\mathrm{B}} T \ln \left(1+e^{-E_{\mathrm{F}, \mathrm{T}=0} / k_{\mathrm{B}} T}\right)$. The frequency-dispersive dielectric function of $\mathrm{SiO}_{2}$ is obtained from Palik ${ }^{46}$.

\section{RESULTS AND DISCUSSION}

The excitation of localized surface plasmon resonances in a DLG nanoribbon array (2L-GNRA) is conceptually shown in Figure 1a. The nanoribbons have width $W$ and period $P$. Scanning electron and atomic force microscope images of a typical 2L-GNRA (Figure $1 \mathrm{~b}$ and 1c) show that the fabricated nanoribbons possess uniform width and well-defined edges. The infrared spectra of the 2L-GNRAs are presented in Figure 1d and are compared against those of single-layer nanoribbons (1L-GNRAs) with identical lateral dimensions. We observe that the extinction peaks produced by DLG have much higher intensity than those produced by SLG. The extinction intensity of 2LGNRAs is approximately three times higher than that of 1L-GNRAs. This is a crucial advantage of DLG, which makes it very useful for infrared plasmonic applications where one of the main challenges is the poor coupling of free-space infrared radiation to the highly subwavelength and strongly confined plasmonic modes of graphene. Interestingly, unlike other techniques that rely on external resonant structures to increase graphene absorption ${ }^{47}$, the enhancement in DLG is intrinsic to the graphene layers and does not limit its frequency range of operation.

In addition to the higher extinction, we observe that 2L-GNRAs resonate at higher frequencies with respect to 1L-GNRAs. Therefore, it is possible to obtain a plasmonic resonance at a specific frequency (wavelength) using wider DLG ribbons instead of narrower SLG ribbons. This is particularly useful from a fabrication perspective because the nanostructuring of ribbons with highly uniform width poses significant challenges, especially at infrared frequencies for which the required ribbon widths are of the order of tens of nanometers. A further important advantage is that the quality factor of the plasmonic resonances in DLG is essentially maintained with a

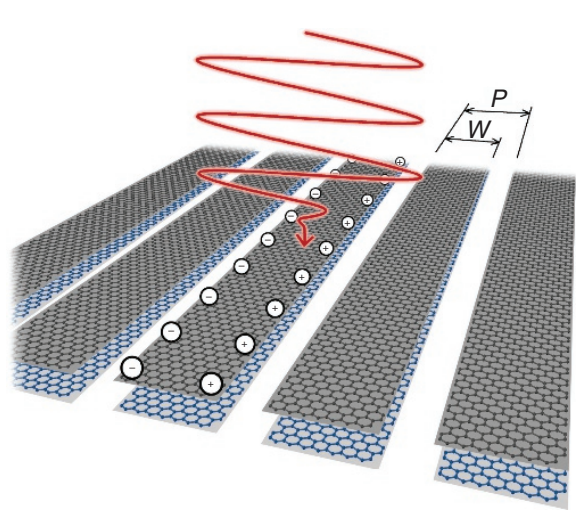

b

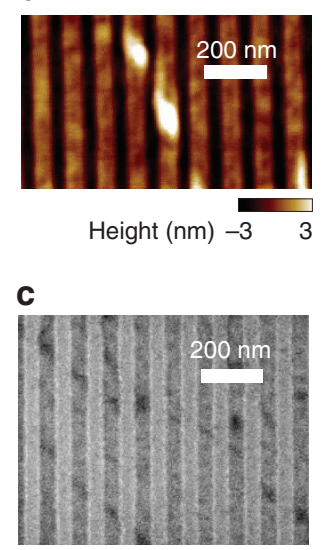

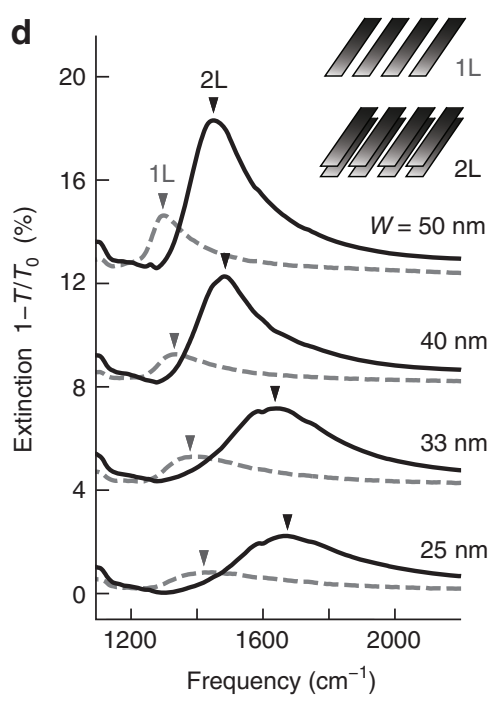

Figure 1 Plasmonic resonances in SLG and DLG nanoribbons. (a) Conceptual representation of the excitation of localized surface plasmon resonances in a 2L-GNRA by illumination with mid-infrared radiation. The top graphene layer is transferred directly over the bottom layer without an interlayer spacer. Graphene nanoribbons are patterned with a width $W$ and periodicity $P$. (b) Surface topography image measured with an atomic force microscope and (c) scanning electron microscope image of one of the fabricated $2 \mathrm{~L}-\mathrm{GNRAs}$ ( $W=50 \mathrm{~nm}, P=105 \mathrm{~nm}$ ). (d) Experimental extinction spectra of $2 \mathrm{~L}-\mathrm{GNRAs}$ (solid curves) with different widths ( $W=25,33,40,50 \mathrm{~nm}$ and $P \approx 2 W$ ). The 2L-GNRA spectra are compared against those of $1 \mathrm{~L}-\mathrm{GNRAs}$ (dashed curves) with identical width and periodicity. The extinction is calculated as $1-T / T_{0}$, where $T$ and $T_{0}$ are the transmission coefficients with and without GNRAs, respectively. 
respect to SLG. This indicates that the carrier scattering rate and mobility in DLG and SLG are similar.

The spectra of 2L-GNRAs do not present any sharp feature superimposed on the plasmonic resonance, indicating that DLG is free of infrared-active phonons in this spectral range. For most applications, such as plasmon-enhanced biosensing, these additional features in the plasmonic response are undesired ${ }^{32}$. In this sense, DLG presents a clear advantage over other related graphene materials such as bilayer graphene or graphene-boron nitride heterostructures, which exhibit infrared-active phonons at 1580 and $1370 \mathrm{~cm}^{-1}$, respectively ${ }^{41,43,48}$. We remark that the DLG used in this work should not be confused with bilayer graphene, in which the atomic alignment of the two graphene layers strongly modifies the electronic band structure and ensues optical properties ${ }^{41,49}$. Because of our fabrication approach, in which we perform two independent transfers of CVD graphene, the interlayer separation is larger than in bilayer graphene and there is no crystallographic alignment; therefore, the plasmonic effects of interlayer tunneling are negligible ${ }^{42}$. Roughness measurements of the bottom graphene layer indicate a minimum average interlayer spacing of $1.0 \mathrm{~nm}$ because of the presence of polymeric residues and graphene wrinkles.
We study next the optical response of the GNRAs when the Fermi level of graphene is electrostatically tuned. The tuning scheme is outlined in Figure 2a and is based on applying a voltage difference across the $\mathrm{SiO}_{2}$ layer to inject or remove electrons from the graphene layers and modify their Fermi level. In contrast to previous work ${ }^{15}$, our stack geometry avoids using isolating spacers between layers and keeps the graphene layers electrically interconnected, allowing them to be simultaneously biased. The physical mechanisms underlying the biasing of MLG are analyzed later in this manuscript. The voltagedependent spectra are shown in Figure $2 \mathrm{~b}$ and $2 \mathrm{c}$ for $1 \mathrm{~L}$ - and 2L-GNRAs, respectively. For 1L-GNRAs, we observe that biasing from $0 \mathrm{~V}$ to negative voltages (up to $-100 \mathrm{~V}$ ) produces a progressive shift of the plasmonic resonance toward higher frequencies and an increase in the resonance intensity. This result is consistent with previous experiments reported by other research groups $^{14,16,21}$ and is a consequence of the initial p-doping of our CVD graphene $\left(E_{\mathrm{F} 0}<0\right)$, for which negative voltages produce an increase in the hole carrier density. The results of biasing 2L-GNRAs with negative biasing voltages show identical behavior as in 1L-GNRAs, that is, a progressive blue shift of the resonance accompanied by an increase in its extinction. A clear advantage of 2L-GNRAs is that for the maximum a

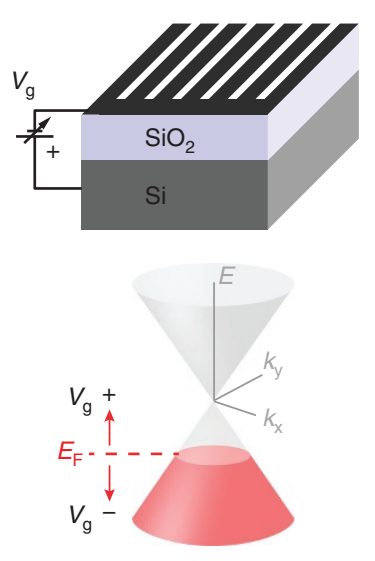

b

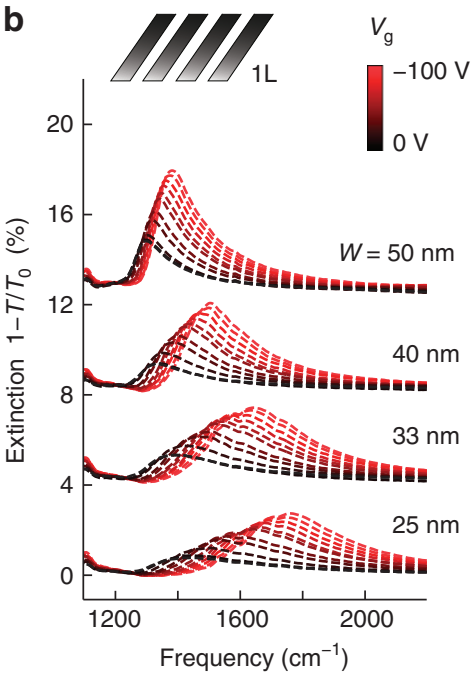

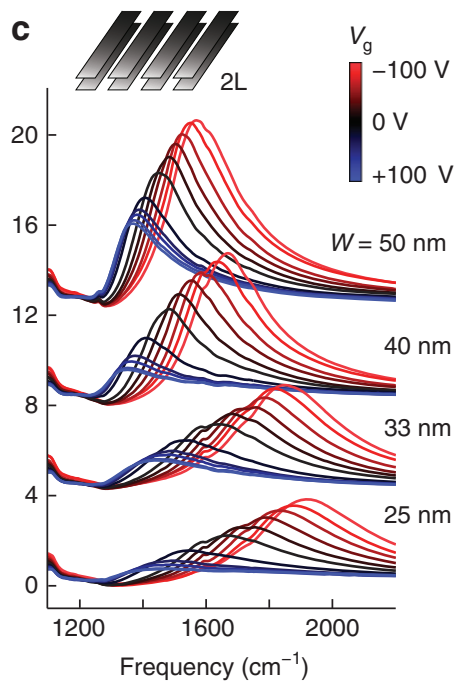

d

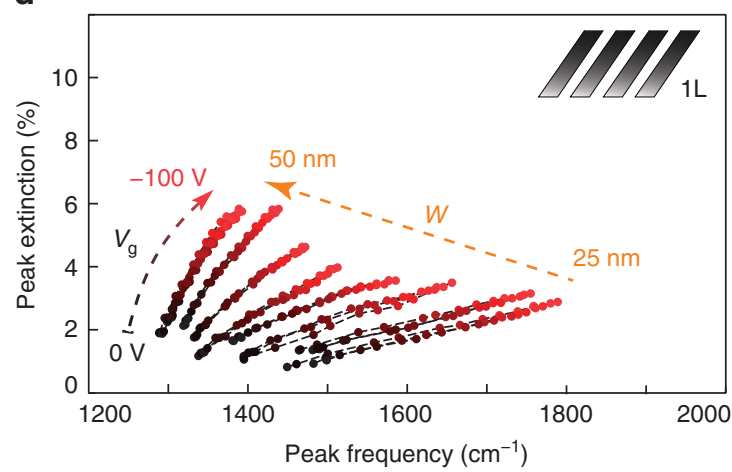

e

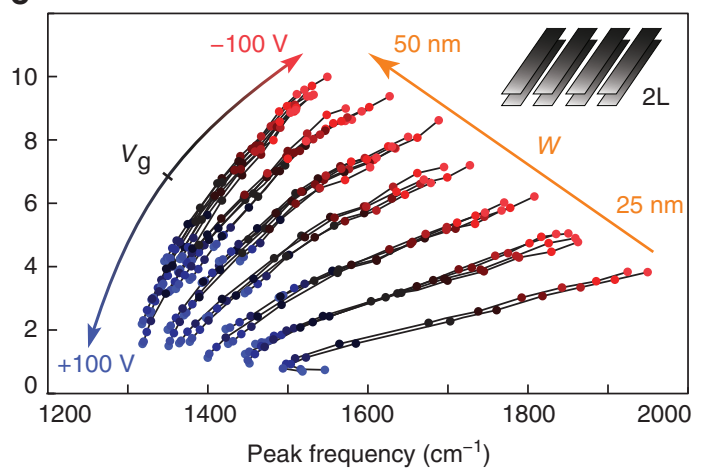

Figure 2 Electrostatic tuning of plasmonic resonances in 1L- and 2L-GNRAs. (a) Electrostatic biasing scheme of graphene nanoribbon arrays. The biasing voltage is applied across the 280-nm-thick $\mathrm{SiO}_{2}$ layer. Negative (positive) voltages shift the Fermi level $\left(E_{\mathrm{F}}\right)$ of our p-doped graphene away from (toward) the Dirac point. (b, c) Experimental extinction spectra of $1 \mathrm{~L}$ - and $2 \mathrm{~L}$-GNRAs for different biasing voltages $V_{\mathrm{g}}$. For $1 \mathrm{~L}$-GNRAs the voltage $V_{\mathrm{g}}$ is varied between $0 \mathrm{~V}$ (black) and $-100 \mathrm{~V}$ (red), whereas for $2 \mathrm{~L}-\mathrm{GNRAs} V_{\mathrm{g}}$ is varied between $+100 \mathrm{~V}$ (blue) and $-100 \mathrm{~V}$ (red). (d, e) Extinction value and spectral position of the measured plasmonic peak in 1L- and 2L-GNRAs for different ribbon widths $W$ and biasing voltages $V_{\mathrm{g}}$. 
negative bias they reach higher frequencies and intensity than 1L-GNRAs with the same lateral dimensions. In addition, while in 1L-GNRAs the use of positive bias voltage quenches the plasmonic resonance, this does not happen in 2L-GNRAs where the much higher extinction of the plasmonic peaks makes it possible to use positive biasing. Figure $2 \mathrm{c}$ shows that positive bias voltages push the plasmonic resonance toward lower frequencies and thus increase the spectral tuning range.

We perform an extended comparison between SLG and DLG in Figure $2 \mathrm{~d}$ and $2 \mathrm{e}$, where we present the spectral position and extinction of the plasmonic peaks for all the fabricated arrays and tested bias voltages. Each marker in the plot corresponds to a specific array and voltage $\left(V_{\mathrm{g}}\right)$, with its horizontal position indicating the resonance frequency and the vertical axis showing the intensity of the resonance peak. At least two identical arrays were fabricated for each value of the width $W$, which correspond to the overlapping lines in the graph, demonstrating excellent repeatability. For a variable voltage $V_{\mathrm{g}}$ there is a linear relationship between the resonance peak intensity and spectral position for both 1L- and 2L-GNRAs. The linear relationship is only broken at the lowest frequencies, where $\mathrm{SiO}_{2}$ is strongly dispersive and its effect is equivalent to a compression of the frequency axis. The comparison between Figure $2 \mathrm{~d}$ and 2e highlights very clearly the advantages of DLG over SLG: higher peak intensity (up to $10 \%$ for $2 \mathrm{~L}$ and $6 \%$ for $1 \mathrm{~L}$ ), higher resonance frequency (up to $1950 \mathrm{~cm}^{-1}$ for $2 \mathrm{~L}$ and $1800 \mathrm{~cm}^{-1}$ for $1 \mathrm{~L}$ ) and wider tuning range (up to $450 \mathrm{~cm}^{-1}$ for $2 \mathrm{~L}$ and $300 \mathrm{~cm}^{-1}$ for $\left.1 \mathrm{~L}\right)$.

To understand the physical mechanisms controlling the plasmonic response of DLG, we perform numerical simulations for a $2 \mathrm{~L}-\mathrm{GNRA}$ composed of two graphene layers separated by a distance $s$ and with Fermi levels $E_{\mathrm{F}}{ }^{(1)}$ and $E_{\mathrm{F}}{ }^{(2)}$ defined relative to their respective Dirac points. In the spectral range of interest, the conductivity of graphene follows the Drude model ${ }^{50,51}$

$$
\sigma=\frac{i e^{2}\left|E_{\mathrm{F}}\right|}{\hbar \pi(\omega+i / \tau)}
$$

Figure 3a shows the evolution of the simulated 2L-GNRA extinction spectra with interlayer separation $s$. In these calculations, we study plasmonic effects for non-varying graphene parameters and exclude, for instance, potential variations of Fermi level induced by changes in the interlayer distance. For large separations $(s>W)$ the 2L-GNRA produces two resonances matching those of the individual layers, that is, the resonance frequency of $1 \mathrm{~L}-$ GNRAs with Fermi levels $E_{\mathrm{F}}^{(1)}$ and $E_{\mathrm{F}}{ }^{(2)}$ (dashed lines). This indicates that for a separation comparable to or larger than the ribbon width, the two graphene layers are electromagnetically uncoupled. As we progressively reduce the interlayer separation $s$, there is an increasing spatial overlap between the near-fields of the two graphene layers and therefore higher electromagnetic coupling ${ }^{52}$. This is evidenced in our simulations by the progressive spectral shift of the two resonances as the separation $s$ approaches zero. The simulated plasmonic response is in perfect agreement with a hybridization model that predicts symmetric and antisymmetric modes with resonance frequencies above and below those from the two individual layers, respectively ${ }^{53-55}$. The simulated charge distribution corresponding to these two modes is shown in Figure $3 \mathrm{~b}$. It is clear that the dipolar modes of each layer have the same polarity for the symmetric mode and opposite polarity for the antisymmetric mode. When the separation $s$ becomes much smaller than the width $W$, the antisymmetric mode is extinguished (that is, it becomes dark) and only the symmetric mode remains. It is this symmetric mode that is detected in our 2L-GNRAs experimental results, for which we measure a higher resonance frequency and extinction than for 1L-GNRAs.

The response of the 2L-GNRA is further analyzed in Figure 3c, where we compare the extinction spectra of 1L-GNRAs (dashed lines) and 2L-GNRAs (solid lines) for an interlayer separation $s$ varying from $s=W$ (red curve) to $s=W / 100$ (blue curve). As expected, when the two layers are uncoupled $(s=W)$ there is nearly perfect overlap between the 2L-GNRA spectrum and that of two independent 1L-GNRAs with Fermi levels $E_{\mathrm{F}}{ }^{(1)}$ and $E_{\mathrm{F}}{ }^{(2)}$. Interestingly, when the two layers are fully coupled $(s \ll W)$ the 2L-GNRA spectrum perfectly matches the spectrum of a 1L-GNRA with an equivalent Fermi level $E_{\mathrm{F}}{ }^{2 \mathrm{~L}}$, given by the sum of the Fermi levels of the individual layers $\left(E_{\mathrm{F}}^{2 \mathrm{~L}}=\left|E_{\mathrm{F}}^{(1)}\right|+\left|E_{\mathrm{F}}(2)\right|\right)^{56-59}$. At first glance, this result is counterintuitive in the context of collective electronic excitations in coupled 2D systems ${ }^{60}$, since one might expect the equivalent carrier density of the two-layer system to be the sum of the carrier densities in each layer $\left(n_{\mathrm{s}}{ }^{(1)}+n_{\mathrm{s}}{ }^{(2)}\right)$, which is not the case here. The explanation for the equivalence between a 2L-GNRA with Fermi levels $E_{\mathrm{F}}{ }^{(1)}, E_{\mathrm{F}}^{(2)}$ and a 1L-GNRA with Fermi level $\left|E_{\mathrm{F}}^{(1)}\right|+\left|E_{\mathrm{F}}{ }^{(2)}\right|$ is that for small separations one can replace the two parallel conductive layers by an equivalent layer having the sum of the two conductivities. For the Drude-model graphene conductivity (Equation (1)), the resulting equivalent conductivity of the two-layer system is

$$
\sigma=\sigma_{1}+\sigma_{2}=\frac{i e^{2}\left(\left|E_{\mathrm{F}}^{(1)}\right|+\left|E_{\mathrm{F}}^{(2)}\right|\right)}{\hbar \pi(\omega+i / \tau)}
$$

This expression corresponds to the Drude conductivity for a graphene layer with Fermi level

$$
E_{\mathrm{F}}^{2 \mathrm{~L}}=\left|E_{\mathrm{F}}^{(1)}\right|+\left|E_{\mathrm{F}}^{(2)}\right|
$$

Accordingly, we define the effective doping of DLG as the sum of the individual doping levels of its two constituent layers. Taking into account the relation between Fermi level and carrier density $\left(E_{\mathrm{F}}=\hbar v_{\mathrm{F}} \sqrt{\pi n_{s}}\right)$, the equivalent carrier density of DLG is

$$
n_{s}^{2 L}=\left|n_{s}^{(1)}\right|+\left|n_{s}^{(2)}\right|+2 \sqrt{\left|n_{s}^{(1)}\right|\left|n_{s}^{(2)}\right|}
$$

It is important to note that, even though the present work focuses on the nanoribbon geometry, the results are equally valid for any arbitrary shape, as long as the separation between the layers is much smaller than the plasmon wavelength and the graphene conductivity follows a Drude model. In this model, the plasmon frequency scales as $E_{\mathrm{F}}^{1 / 2}$ with the Fermi energy, and, consequently, the 2L-GNRA has a higher resonance frequency than a 1L-GNRA with the same total carrier density $\left(n_{s}^{(1)}+n_{s}^{(2)}\right)$, reaching a frequency increase factor of $2^{1 / 4}$ (that is, $20 \%$ higher) when the carriers are equally distributed among the two layers.

This hybridization model can easily be extended to a higher number of layers. An analogous study for a three-layer graphene structure (Supplementary Fig. S1) shows that the different polarities of the dipolar modes on each graphene layer give rise to multiple modes. As in the 2L-GNRA, when the interlayer separation $s$ approaches zero, all of the modes become dark, except the so-called bonding mode in which all layers have the same polarity. For small separations $(s \ll W)$ the spectrum of the multilayer structure matches that of a 1LGNRA, adding up the Fermi levels of all layers $\left(E_{\mathrm{F}}^{N L}=\sum_{i=1}^{N}\left|E_{\mathrm{F}}^{(i)}\right|\right)$ or equivalently having a carrier density $n_{s}^{N L}=\left(\sum_{i=1}^{N} \sqrt{\left|n_{s}^{(i)}\right|}\right)^{2}$. Therefore, increasing the number of layers is a practical approach to create graphene structures with higher equivalent doping. However, a word 

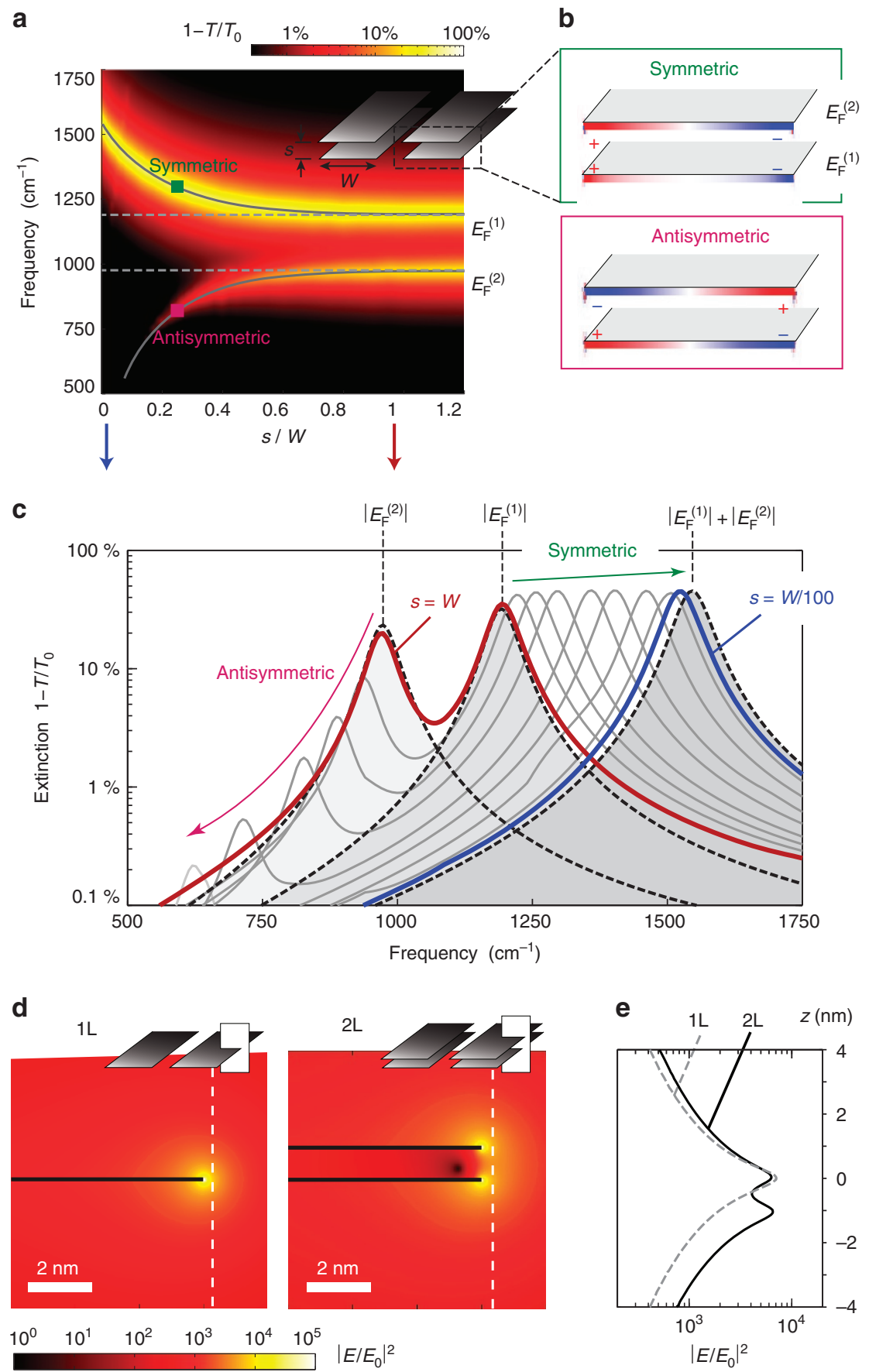

Figure 3 Physical mechanisms and hybridization in 2L-GNRAs. (a) Simulated extinction coefficient of a 2L-GNRA for a variable interlayer separation s. The two graphene layers have Fermi levels $\left|E_{\mathrm{F}}^{(1)}\right|=0.3 \mathrm{eV}$ and $\left|E_{\mathrm{F}}{ }^{(2)}\right|=0.2 \mathrm{eV}$, relaxation time $\tau=100 \mathrm{fs}$, the width of ribbons is $W=40 \mathrm{~nm}$ and are embedded in a dielectric with refractive index $n=1.4$. Solid curves represent the resonance frequencies of the $2 \mathrm{~L}-\mathrm{GNRA}$ and dashed curves stand for the resonance of each individual layer, which correspond to the resonance of a $1 L-G N R A$ with Fermi level $\left|E_{F}^{(1)}\right|$ or $\left|E_{F}^{(2)}\right|$. (b) Electric charge distribution across the ribbons for the 2L-GNRA with $s=W / 4$ at the two resonance frequencies indicated in the figure. The high- (low-) resonance corresponds to a symmetric (antisymmetric) mode. (c) Simulated extinction spectra of the 2L-GNRA (solid curves) for an interlayer separation varying from $s=W$ (red curve) to $s=W / 100$ (blue curve). Dashed curves represent the extinction spectra of $1 \mathrm{~L}-\mathrm{GNRAs}$ with Fermi levels $\left|E_{\mathrm{F}}{ }^{(1)}\right|,\left|E_{\mathrm{F}}(2)\right|$ and $\left|E_{\mathrm{F}}{ }^{(1)}\right|+\left|E_{\mathrm{F}}{ }^{(2)}\right|$. (d) Field enhancement distribution at resonance frequency for $1 \mathrm{~L}-\mathrm{GNRA}\left(\left|E_{\mathrm{F}}\right|=0.3 \mathrm{eV}\right)$ and for the symmetric mode of a $2 \mathrm{~L}-\mathrm{GNRA}\left(\left|E_{\mathrm{F}}{ }^{(1)}\right|=0.3 \mathrm{eV},\left|E_{\mathrm{F}}{ }^{(2)}\right|=0.2 \mathrm{eV}\right)$ with a separation $s=1 \mathrm{~nm}$. The field is presented for the cross-section indicated in the inset. (e) Field enhancement for the 1L-and 2L-GNRAs along the white dashed line in subfigure $\mathrm{d}$.

of caution has to be given: the higher effective doping of DLG does not extend the spectral region that is free from interband transitions $\left(\hbar \omega<2\left|E_{\mathrm{F}}^{(i)}\right|\right)^{50}$, a condition that is required in order to have longlived plasmons that are unaffected by Landau damping.
A study of the plasmonic field distributions is presented in Figure 3d, where we compare the near fields in 1L- and 2L-GNRAs. We observe that both structures display field distributions corresponding to dipolar modes with intense near-field hotspots at the graphene 
a

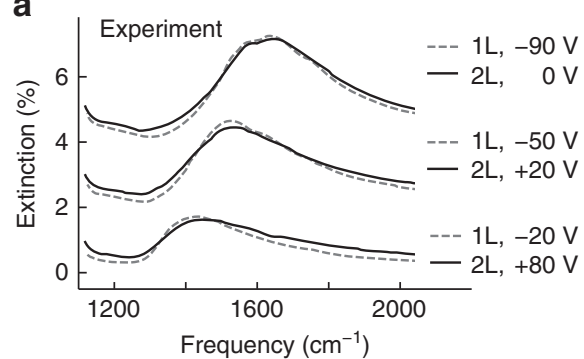

b

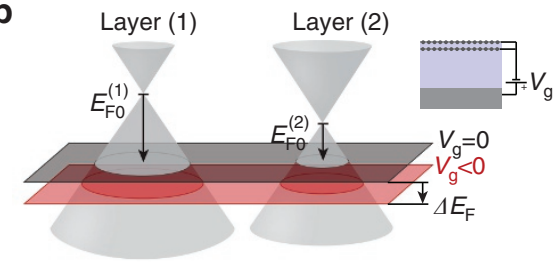

C

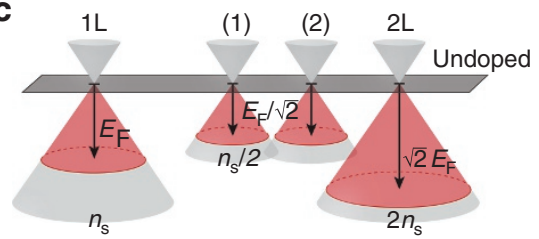

d

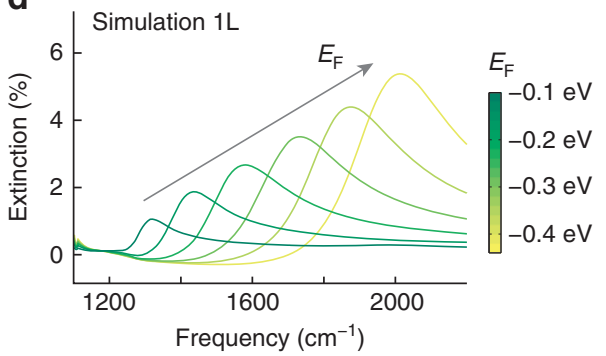

e

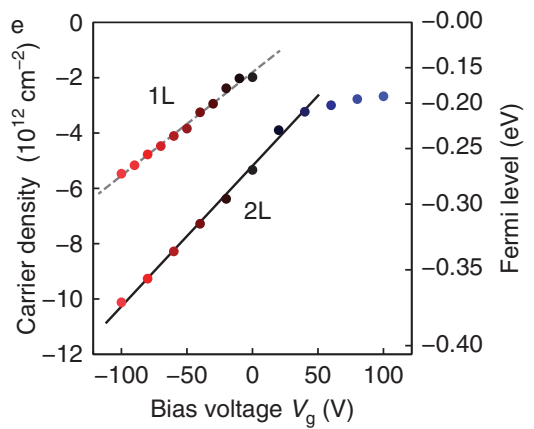

Figure 4 Equivalent doping and enhanced tunability of DLG. (a) Comparison between the experimental extinction spectra of 1L- and 2L-GNRAs with $W=33 \mathrm{~nm}$ and specific pairs of bias voltage $V_{\mathrm{g}}$. (b) Band diagram representation of a multilayer graphene stack composed of two layers that are electrically interconnected. The black/red planes represent the Fermi level before/after biasing. (c) Band diagram representation for initially undoped graphene layers being biased. The Fermi levels and carrier densities are represented for SLG (1L), DLG (2L) and its two individual layers (1) and (2). (d) Simulated extinction spectra of the $1 \mathrm{~L}$-GNRA with $W=33 \mathrm{~nm}$, graphene relaxation time $\tau=15 \mathrm{fs}$ and varying Fermi level $E_{\mathrm{F}}$. (e) Equivalent carrier density and Fermi level of the $1 \mathrm{~L}$ - and 2L-GNRA extracted from experimental spectra. The dashed and solid curves represent a linear fit of the equivalent carrier density for the $1 \mathrm{~L}-$ and 2L-GNRA, respectively.

nanoribbon edges. Furthermore, in Figure $3 e$ we present the field intensity profile perpendicularly to the nanoribbons close to the ribbon edge ( $0.3 \mathrm{~nm}$ distance), thus avoiding any inaccuracy introduced by classical electromagnetic computations near sharp boundaries. We observe that the field distribution, confinement and optical enhancement in DLG do not differ significantly from those in SLG.

The plasmonic equivalence between SLG and DLG can be experimentally demonstrated by comparing the measured spectra of the respective nanoribbon arrays. Figure $4 \mathrm{a}$ shows the extinction spectra of 1L-GNRA and 2L-GNRA for specific pairs of biasing voltages. We can clearly see that the spectrum of the 2L-GNRA for a given voltage is in excellent agreement with that of the 1L-GNRA for a significantly lower (more negative) voltage. These results provide experimental confirmation that a 2L-GNRA is equivalent to a 1L-GNRA with a different Fermi level. The change of the equivalent Fermi level with bias voltage in MLG is investigated next.

We consider a stack of $N$ graphene layers having different levels of initial doping $\left(E_{\mathrm{F} 0}^{(i)}\right.$ for the i-th layer), being electrically interconnected to be simultaneously biased and having an interlayer separation $s$ tending to zero. In this case, the Dirac cones of the interconnected layers shift along the energy axis; therefore, the Fermi levels of all layers are aligned ${ }^{61-64}$, as shown in Figure $4 \mathrm{~b}$ for two layers. Increasing the bias voltage $V_{\mathrm{g}}$ shifts the overall Fermi level and as a result the injected carriers are redistributed among the different layers proportionally to their doping level. The Fermi level of each layer after applying a bias $\left(E_{\mathrm{F}}^{(i)}\right)$ is calculated by assuming that the Fermi-level variation is the same for all layers and that the extra carriers in the graphene layers $\left(n_{s}^{(i)}-n_{s 0}^{(i)}\right)$ add up to the total charge in a capacitor with surface capacitance $C_{\mathrm{Ox}}$ :

$$
\begin{gathered}
E_{\mathrm{F}}^{(i)}-E_{\mathrm{F} 0}^{(i)}=E_{\mathrm{F}}^{(1)}-E_{\mathrm{F} 0}^{(1)} \quad i=1 . . N \\
\sum_{i=1}^{N} n_{s}^{(i)}-n_{s 0}^{(i)}=C_{\mathrm{ox}} V_{\mathrm{g}} / e
\end{gathered}
$$

where the Fermi level of each layer $E_{\mathrm{F}}^{(i)}$ is defined as relative to its corresponding Dirac point. This set of second-order equations $\left(E_{\mathrm{F}} \sim \sqrt{n_{s}}\right)$ has analytical solution in the case where all graphene layers have the same type of doping ( $\mathrm{p}$ or $\mathrm{n}$ ). After some calculations, the variation of Fermi level $\left(\Delta E_{\mathrm{F}}\right.$ in Figure $\left.4 \mathrm{~b}\right)$ can be written in the simple form (see the Supplementary Information for a detailed derivation)

$$
E_{\mathrm{F}}^{(i)}-E_{\mathrm{F} 0}^{(i)}=\left[\left(\frac{E_{\mathrm{F} 0}^{N L}}{N}\right)^{2} \pm \frac{1}{N} A V_{\mathrm{g}}\right]^{1 / 2}-\frac{E_{\mathrm{F} 0}^{N L}}{N}
$$

where $A=C_{\mathrm{ox}} \hbar^{2} v_{\mathrm{F}}^{2} \pi / e$ and the plus/minus sign accounts for the doping type of the graphene layers ( $\mathrm{p}$ - or $\mathrm{n}$-doped). Notice that the Fermi level variation does not depend on the individual doping levels of the graphene layers $\left(E_{F 0}^{(i)}\right)$, as one might initially expect. Instead, it depends only on the sum of the initial levels $\left(E_{\mathrm{F} 0}^{N L}=\sum_{i=1}^{N} E_{\mathrm{F} 0}^{(i)}\right)$, which we have previously shown to correspond to the equivalent Fermi level of the MLG. Strictly speaking, the equivalent Fermi level corresponds to the sum of the absolute doping levels $\left(E_{\mathrm{F} 0}^{N L}=\sum_{i=1}^{N}\left|E_{\mathrm{F} 0}^{(i)}\right|\right)$; however, the expression without absolute values can be used when all layers 
share a common doping type. Next, we calculate the equivalent Fermi level of the biased MLG $\left(E_{\mathrm{F}}^{N L}\right)$ by adding Equation (7) for all layers

$$
\left[E_{\mathrm{F}}^{N L}\right]^{2}-\left[E_{\mathrm{F} 0}^{N L}\right]^{2}= \pm N A V_{\mathrm{g}}
$$

or in terms of the equivalent carrier density $n_{s}^{N L}=\left(\sum_{i=1}^{N} \sqrt{\left|n_{s}^{(i)}\right|}\right)^{2}$,

$$
n_{s}^{N L}-n_{s 0}^{N L}= \pm N C_{\mathrm{ox}} V_{\mathrm{g}} / e
$$

This simple result shows that the increase in the equivalent carrier density of MLG is enhanced with respect to SLG by a factor corresponding to the number of layers $N$. This insight is of crucial importance for plasmonic applications, since it shows that MLG provides broader tunability than SLG. In particular, the biasing of DLG can be twice as effective as in SLG, meaning a twofold increase in the equivalent carrier density for the same bias voltage. This effect can be readily understood for the case of undoped graphene layers $\left(E_{\mathrm{F} 0}^{(1)}=E_{\mathrm{F} 0}^{(2)}=0\right)$, where the carriers split equally between the two layers $\left(n_{s}^{(1)}=n_{s}^{(2)}=\frac{1}{2} n_{s}^{1 L}\right)$ and from Equation (4) the equivalent DLG doping is $n_{s}^{2 L}=2 n_{s}^{1 L}$ (Figure 4c). Our simple model provides a clear and intuitive description of the effects of electrostatic tuning over the plasmonic response of MLG; however, more elaborate theoretical models may be required for accurate calculations of charge redistribution in structures with a large number of graphene layers ${ }^{65-67}$. The biasing effectiveness can be degraded if carriers are redistributed differently between the two graphene layers. However, the impact of a moderate charge redistribution over the equivalent carrier density is relatively low. For instance, if an undoped layer receives four times as many carriers as a second undoped layer, the equivalent carrier density according to Equation (4) is reduced by just $10 \%$ with respect to an even distribution of charges.

To experimentally validate the enhanced tuning capabilities of MLG, we extract the equivalent Fermi level of our 2L-GNRA for the different voltages and compare it against that of the 1L-GNRA. Specifically, we extract the Fermi level by comparison with electromagnetic simulations. In Figure $4 \mathrm{~d}$ we show the simulated extinction spectra of our 1L-GNRAs for different Fermi levels. We observe that the spectral lineshapes are in good agreement with the experimental data. Since the resonance frequency shifts monotonously with the Fermi level, we can use the simulations to connect these two parameters and extract the Fermi level from the experimental data. Figure $4 \mathrm{e}$ presents the extracted carrier densities and Fermi levels of SLG $\left(E_{\mathrm{F}}^{1 \mathrm{~L}} n_{\mathrm{s}}^{1 \mathrm{~L}}\right)$ and DLG $\left(E_{\mathrm{F}}^{2 L}=\left|E_{\mathrm{F}}^{(1)}\right|+\left|E_{\mathrm{F}}^{(2)}\right|\right.$, $n_{s}^{2 L}=\left[\sqrt{\left|n_{s}^{(1)}\right|}+\sqrt{\left|n_{s}^{(2)}\right|}\right]^{2}$. As expected, the equivalent doping level in DLG is significantly higher than in SLG, confirming that DLG is equivalent to highly doped SLG. We observe two distinct behaviors in the DLG carrier density for voltages $V_{\mathrm{g}}$ above or below $+40 \mathrm{~V}$. For high positive voltages, we observe a saturation of the DLG equivalent carrier density. This saturation is caused by the Fermi level (red plane in Figure $4 \mathrm{~b}$ ) crossing the Dirac point of one of the layers, resulting in two layers with opposite doping types (see Supplementary Fig. S3).

Next, we focus on voltages below $+40 \mathrm{~V}$, which correspond to both graphene layers being $\mathrm{p}$-doped. This is the relevant voltage range for plasmonics, since it corresponds to a high doping. In this range, the equivalent carrier density varies linearly with the applied voltage, in agreement with our theoretical calculations (see Equation (7)). This linear relationship is well known for SLG, but is observed here for the first time in DLG. More importantly, the variation of the equivalent carrier density with the bias voltage $\left(\mathrm{d} n_{\mathrm{s}} / \mathrm{d} V_{\mathrm{g}}\right)$ in DLG is $36 \%$ higher than in SLG. This variation, despite being smaller than the theoretical value, confirms that biasing is more effective in MLG structures than in SLG. It is important to emphasize that the improved biasing is not caused by a larger amount of total injected carriers (which depends only on the bias voltage and the gating layer capacitance); instead, it is due to the redistribution of these carriers over multiple plasmonically coupled layers. Finally, it is clear that stacking multiple graphene layers is a powerful approach to enhance the tunability of graphene plasmonic devices.

\section{CONCLUSIONS}

In conclusion, we have shown that DLG, readily fabricated by two successive transfers of CVD graphene, provides significant advantages over SLG for plasmonic applications. DLG operates as a SLG with an equivalent higher doping and serves as a plasmonic platform for higher frequency resonances, with stronger intensity and wider tuning ranges. These results can be further generalized to MLG structures with more than two layers, potentially yielding even better performances. Since high doping and broad tunability are critical requirements for graphene to succeed in plasmonic applications, we believe that the combination of multiple graphene layers opens very promising pathways toward novel reconfigurable nanophotonic devices.

\section{CONFLICT OF INTEREST}

The authors declare no conflict of interest.

\section{ACKNOWLEDGEMENTS}

The research leading to these results has received funding from the European Union Seventh Framework Programme under grant agreements no. 625673 GRYPHON, no. 604391 and from European Union H2020 Programme under grant agreement no. 696656 Graphene Flagship. We acknowledge financial support from the Swiss National Science Foundation through project no. 133583, NATO's Public Diplomacy Division in the framework of 'Science for Peace', European Union's Horizon 2020 research and innovation program under grant agreement no. 644956, Fundació Privada Cellex, AGAUR 2014 SGR 1400 and 1623, the Spanish Ministry of Economy and Competitiveness (grants SEV-2015-0522 and MAT2014-59096-P), and the 'Fondo Europeo de Desarrollo Regional' (FEDER) through grant TEC2013-46168-R. We also acknowledge École Polytechnique Fédérale de Lausanne and Center of MicroNano Technology for nanofabrication.

1 Polman A. Plasmonics applied. Science 2008; 322: 868-869.

2 Maier SA. Plasmonics: Fundamentals and Applications. Springer: USA, 2007.

3 Anker JN, Hall WP, Lyandres O, Shah NC, Zhao J et al. Biosensing with plasmonic nanosensors. Nat Mater 2008; 7: 442-453.

4 Yanik AA, Huang M, Kamohara O, Artar A, Geisbert TW et al. An optofluidic nanoplasmonic biosensor for direct detection of live viruses from biological media. Nano Lett 2010; 10: 4962-4969.

5 Knight MW, Sobhani H, Nordlander P, Halas NJ. Photodetection with active optical antennas. Science 2011; 332: 702-704.

6 Atwater HA, Polman A. Plasmonics for improved photovoltaic devices. Nat Mater 2010; 9: 205-213.

7 Hou WB, Cronin SB. A review of surface plasmon resonance-enhanced photocatalysis. Adv Funct Mater 2013; 23: 1612-1619.

8 Jablan M, Buljan H, Soljacic M. Plasmonics in graphene at infrared frequencies. Phys Rev B 2009; 80: 245435.

9 García de Abajo FJ. Graphene plasmonics: challenges and opportunities. ACS Photonics 2014; 1: 135-152.

10 Geim AK, Novoselov KS. The rise of graphene. Nat Mater 2007; 6: 183-191.

11 Grigorenko AN, Polini M, Novoselov KS. Graphene plasmonics. Nat Photonics 2012; 6: 749-758.

12 Low T, Avouris P. Graphene plasmonics for terahertz to mid-infrared applications. ACS Nano 2014; 8: 1086-1101. 
13 Fei Z, Andreev GO, Bao WZ, Zhang LM, McLeod AS et al. Infrared nanoscopy of dirac plasmons at the graphene- $\mathrm{SiO}_{2}$ interface. Nano Lett 2011; 11: 4701-4705.

14 Ju L, Geng BS, Horng J, Girit C, Martin M et al. Graphene plasmonics for tunable terahertz metamaterials. Nat Nanotechnol 2011; 6: 630-634.

15 Yan HG, Li XS, Chandra B, Tulevski G, Wu YQ et al. Tunable infrared plasmonic devices using graphene/insulator stacks. Nat Nanotechnol 2012; 7: 330-334.

16 Brar VW, Jang MS, Sherrott M, Lopez JJ, Atwater HA. Highly confined tunable midinfrared plasmonics in graphene nanoresonators. Nano Lett 2013; 13: 2541-2547.

17 Yan HG, Low T, Zhu WJ, Wu YQ, Freitag M et al. Damping pathways of mid-infrared plasmons in graphene nanostructures. Nat Photonics 2013; 7: 394-399.

$18 \mathrm{Hu} \mathrm{H}$, Zhai F, Hu DB, Li ZJ, Bai B et al. Broadly tunable graphene plasmons using an ion-gel top gate with low control voltage. Nanoscale 2015; 7: 19493-19500.

19 Yang XX, Kong XT, Bai B, Li ZJ, Hu H et al. Substrate phonon-mediated plasmon hybridization in coplanar graphene nanostructures for broadband plasmonic circuits. Small 2014; 11: 591-596.

20 Nikitin AY, Guinea F, Garcia-Vidal FJ, Martin-Moreno L. Surface plasmon enhanced absorption and suppressed transmission in periodic arrays of graphene ribbons. Phys Rev $B$ 2012; 85: 081405.

21 Fang ZY, Thongrattanasiri S, Schlather A, Liu Z, Ma LL et al. Gated tunability and hybridization of localized plasmons in nanostructured graphene. ACS Nano 2013; 7: 2388-2395.

22 Fang ZY, Wang YM, Schlather AE, Liu Z, Ajayan PM et al. Active tunable absorption enhancement with graphene nanodisk arrays. Nano Lett 2013; 14: 299-304.

23 Zhu XL, Wang WH, Yan W, Larsen MB, Bøggild P et al. Plasmon-phonon coupling in large-area graphene dot and antidot arrays fabricated by nanosphere lithography. Nano Lett 2014; 14: 2907-2913.

24 Chen JN, Badioli M, Alonso-González P, Thongrattanasiri S, Huth F et al. Optical nanoimaging of gate-tunable graphene plasmons. Nature 2012; 487: 77-81.

25 Fei Z, Rodin AS, Andreev GO, Bao W, McLeod AS et al. Gate-tuning of graphene plasmons revealed by infrared nano-imaging. Nature 2012; 487: 82-85.

26 Kim J, Son H, Cho DJ, Geng BS, Regan W et al. Electrical control of optical plasmon resonance with graphene. Nano Lett 2012; 12: 5598-5602.

27 Liu PQ, Luxmoore IJ, Mikhailov SA, Savostianova NA, Valmorra F et al. Highly tunable hybrid metamaterials employing split-ring resonators strongly coupled to graphene surface plasmons. Nat Commun 2015; 6: 8969.

28 Mousavi SH, Kholmanov I, Alici KB, Purtseladze D, Arju N et al. Inductive tuning of fano-resonant metasurfaces using plasmonic response of graphene in the mid-infrared. Nano Lett 2013; 13: 1111-1117.

29 Yao Y, Kats MA, Genevet P, Yu NF, Song Y et al. Broad electrical tuning of grapheneloaded plasmonic antennas. Nano Lett 2013; 13: 1257-1264.

30 Koppens FHL, Chang DE, García de Abajo FJ. Graphene plasmonics: a platform for strong light-matter interactions. Nano Lett 2011; 11: 3370-3377.

31 Woessner A, Lundeberg MB, Gao YD, Principi A, Alonso-González P et al. Highly confined low-loss plasmons in graphene-boron nitride heterostructures. Nat Mater 2015; 14: 421-425.

32 Rodrigo D, Limaj O, Janner D, Etezadi D, García de Abajo FJ et al. Mid-infrared plasmonic biosensing with graphene. Science 2015; 349: 165-168.

33 Sensale-Rodriguez B, Yan RS, Kelly MM, Fang T, Tahy K et al. Broadband graphene terahertz modulators enabled by intraband transitions. Nat Commun 2012; 3: 780

34 Emani NK, Chung T-F, Kildishev AV, Shalaev VM, Chen YP et al. Electrical modulation of fano resonance in plasmonic nanostructures using graphene. Nano Lett 2013; 14: 78-82.

35 Lee SH, Choi M, Kim T-T, Lee S, Liu M et al. Switching terahertz waves with gatecontrolled active graphene metamaterials. Nat Mater 2012; 11: 936-941.

36 Vakil A, Engheta N. Transformation optics using graphene. Science 2011; 332 : 1291-1294.

37 Fallahi A, Perruisseau-Carrier J. Design of tunable biperiodic graphene metasurfaces. Phys Rev B 2012; 86: 195408.

38 Ding J, Arigong B, Ren H, Zhou M, Shao J et al. Tuneable complementary metamaterial structures based on graphene for single and multiple transparency windows. Sci Rep 2014; 4: 6128

39 Thongrattanasiri S, Koppens FHL, García de Abajo FJ. Complete optical absorption in periodically patterned graphene. Phys Rev Lett 2012; 108: 047401.

40 Yao Y, Shankar R, Kats MA, Song Y, Kong J et al. Electrically tunable metasurface perfect absorbers for ultrathin mid-infrared optical modulators. Nano Lett 2014; 14: 6526-6532.

41 Yan HG, Low T, Guinea F, Xia FN, Avouris P. Tunable phonon-induced transparency in bilayer graphene nanoribbons. Nano Lett 2014; 14: 4581-4586.
42 Fei Z, Iwinski EG, Ni GX, Zhang LM, Bao W et al. Tunneling plasmonics in bilayer graphene. Nano Lett 2015; 15: 4973-4978.

43 Brar VW, Jang MS, Sherrott M, Kim S, Lopez JJ et al. Hybrid surface-phonon-plasmon polariton modes in graphene/monolayer h-BN heterostructures. Nano Lett 2014; 14 : 3876-3880.

44 Emani NK, Wang D, Chung T-F, Prokopeva LJ, Kildishev AV et al. Plasmon resonance in multilayer graphene nanoribbons. Laser Photonics Revs 2015; 9: 650-655.

45 Farmer DB, Rodrigo D, Low T, Avouris P. Plasmon-plasmon hybridization and bandwidth enhancement in nanostructured graphene. Nano Lett 2015; 15: 2582-2587.

46 Palik ED. Handbook of Optical Constants of Solids. Academic Press: Amsterdam, The Netherland, 1998.

47 Jang MS, Brar VW, Sherrott MC, Lopez JJ, Kim L et al. Tunable large resonant absorption in a midinfrared graphene Salisbury screen. Phys Rev B 2014; 90: 165409.

48 Kumar A, Low T, Fung KH, Avouris P, Fang NX. Tunable light-matter interaction and the role of hyperbolicity in graphene-hBN system. Nano Lett 2015; 15: 3172-3180.

49 McCann E. Asymmetry gap in the electronic band structure of bilayer graphene. Phys Rev $B$ 2006; 74: 161403.

$50 \mathrm{Li} Z$ Q, Henriksen EA, Jiang Z, Hao Z, Martin MC et al. Dirac charge dynamics in graphene by infrared spectroscopy. Nat Phys 2008; 4: 532-535.

51 Horng J, Chen C-F, Geng BS, Girit C, Zhang YB et al. Drude conductivity of Dirac fermions in graphene. Phys Rev B 2011; 83: 165113.

52 Wang B, Zhang X, Yuan XC, Teng JH. Optical coupling of surface plasmons between graphene sheets. Appl Phys Lett 2012; 100: 131111.

53 Prodan E, Radloff C, Halas NJ, Nordlander P. A hybridization model for the plasmon response of complex nanostructures. Science 2003; 302: 419-422.

54 Christensen J, Manjavacas A, Thongrattanasiri S, Koppens FHL, García de Abajo FJ. Graphene plasmon waveguiding and hybridization in individual and paired nanoribbons. ACS Nano 2012; 6: 431-440.

55 Yu RW, Alaee R, Lederer F, Rockstuhl C. Manipulating the interaction between localized and delocalized surface plasmon-polaritons in graphene. Phys Rev B 2014; 90: 085409 .

56 Hwang EH, Das Sarma S. Plasmon modes of spatially separated double-layer graphene. Phys Rev B 2009; 80: 205405.

57 Rodrigo D, Low T, Farmer DB, Altug H, Avouris P. Plasmon coupling in extended structures: graphene superlattice nanoribbon arrays. Phys Rev B 2016; 93: 125407.

58 Van Tuan D, Khanh NQ. Plasmon modes of double-layer graphene at finite temperature. Phys E 2013; 54: 267-272.

59 Svintsov D, Vyurkov V, Ryzhii V, Otsuji T. Voltage-controlled surface plasmon-polaritons in double graphene layer structures. J Appl Phys 2013; 113: 053701.

60 Das Sarma S, Madhukar A. Collective modes of spatially separated, two-component, two-dimensional plasma in solids. Phys Rev B 1981; 23: 805-815.

61 Cheianov VV, Fal'ko V, Altshuler BL. The focusing of electron flow and a veselago lens in graphene p-n junctions. Science 2007; 315: 1252-1255.

62 Farmer DB, Lin Y-M, Afzali-Ardakani A, Avouris P. Behavior of a chemically doped graphene junction. Appl Phys Lett 2009; 94: 213106.

63 Low T, Hong S, Appenzeller J, Datta S, Lundstrom MS. Conductance asymmetry of graphene p-n junction. IEEE Trans Electron Dev 2009; 56: 1292-1299.

64 Xu XD, Gabor NM, Alden JS, van der Zande AM, McEuen PL. Photo-thermoelectric effect at a graphene interface junction. Nano Lett 2010; 10: 562-566.

65 Guinea F. Charge distribution and screening in layered graphene systems. Phys Rev $B$ 2007; 75: 235433

66 Kuroda MA, Tersoff J, Martyna GJ. Nonlinear screening in multilayer graphene systems. Phys Rev Lett 2011; 106: 116804.

67 Kuroda MA, Tersoff J, Nistor RA, Martyna GJ. Optimal thickness for charge transfer in multilayer graphene electrodes. Phys Rev App/ 2014; 1: 014005.

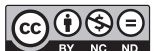

This work is licensed under a Creative Commons AttributionNonCommercial-NoDerivs 4.0 International License. The images or other third party material in this article are included in the article's Creative Commons license, unless indicated otherwise in the credit line; if the material is not included under the Creative Commons license, users will need to obtain permission from the license

holder to reproduce the material. To view a copy of this license, visit http:// creativecommons.org/licenses/by-nc-nd/4.0/

(C) The Author(s) 2017

Supplementary Information for this article can be found on the Light: Science \& Applications' website (http://www.nature.com/lsa). 